\title{
Implementación de una Estrategia Didáctica Basada en Tecnologías de la Información y la Comunicación en Prevención del Embarazo en Adolescentes para el Nivel Secundaria del Sector Público
}

\author{
Implementation of a Teaching Strategy Based on Information and Communication Technologies \\ in Adolescent Pregnancy Prevention for the Secondary Level of the Public Sector
}

E. Bolaños-Rodríguez ${ }^{a}$, L.G. Gómez-Oropeza ${ }^{b}$ and E. Lezama-León ${ }^{c}$

\begin{abstract}
:
The paper presents a proposal for the development of a didactic strategy based on Information and Communication Technologies (ICT) in adolescent pregnancy prevention for secondary level in the public sector. It is aimed at male and female students aged 12 to 15 years through the execution of a workshop in which information about family planning methods, sexually transmitted infections and obstetric risk factors is provided. The use of technology is implemented through visual and auditory multimedia tools, learning objects, web pages, digital presentations, didactic sequences and evaluation techniques. The NEO educational platform is used, where the workshop is addressed, active means of communication are used, such as the forum and chat. The methodology is based on the instructional design of Jerold Kemp, which is useful both for the development of teaching units and for the entire course, providing a framework in which all types of activities and creative experiences can be anticipated. The main results obtained show the interest and motivation of the adolescents in the workshop regarding the issue of pregnancy prevention when the technologies are used for educational purposes, considering the opportune intervention of the teacher shown through the teaching strategies with the incorporation of the TIC.
\end{abstract}

Keywords:

Didactic strategy, Information and Communication Technologies, Pregnancy, Adolescent and NEO Platform

\section{Resumen:}

El trabajo presenta una propuesta de desarrollo de estrategia didáctica basada en Tecnologías de la Información y la Comunicación (TIC) en prevención del embarazo en adolescentes para nivel secundaria del sector público. Está enfocado para estudiantes del sexo masculino y femenino de 12 a 15 años a través de la ejecución de un taller en el cual se proporciona información sobre métodos de planificación familiar, infecciones de transmisión sexual y factores de riesgo obstétrico. Se implementa el uso de la tecnología por medio de herramientas multimedia visual y auditiva, objetos de aprendizaje, páginas web, presentaciones digitales, secuencias didácticas y técnicas de evaluación. Se utiliza la plataforma educativa NEO, donde se aborda el taller, se emplean medios activos de comunicación como son el foro y el chat. La metodología está basada en el diseño instruccional de Jerold Kemp, el cual es útil tanto para el desarrollo de unidades didácticas como para el curso completo, proporciona un marco en el que pueden anticiparse todos los tipos de actividades y experiencias creativas. Los principales resultados obtenidos evidencian el interés y la motivación de los adolescentes en el taller respecto al tema de prevención de embarazo cuando se emplean las tecnologías con fines educativos, considerando la oportuna intervención del docente mostradas a través de las estrategias didácticas con la incorporación de las TIC.

Palabras Clave:

Estrategia didáctica, Tecnologías de la Información y la Comunicación, Embarazo, Adolescente y Plataforma NEO

\footnotetext{
a Escuela Superior de Tizayuca, Universidad Autónoma del Estado de Hidalgo, México, Km. 2.5 de la Carretera Federal Tizayuca-Pachuca, 43802, Tizayuca, Hidalgo. E-mail: ebolanos@uaeh.edu.mx

b Instituto de Ciencias Básicas e Ingeniería Universidad Autónoma del Estado de Hidalgo, México, Avenida San Judas Tadeo 132, Carboneras, 42183, Mineral de la Reforma, Hidalgo. E-mail: promotstizayuca@gmail.com

${ }^{\mathrm{C}}$ Escuela Superior de Tizayuca, Universidad Autónoma del Estado de Hidalgo, México, Km. 2.5 de la Carretera Federal Tizayuca-Pachuca, 43802, Tizayuca, Hidalgo. E-mail: evangeli@uaeh.edu.mx
} 


\section{Introducción}

La falta de apoyo, información y orientación en materia de salud reproductiva y educación sexual para niñas y adolescentes, ha ocasionado que el índice de embarazos en esta etapa de la vida se considere un problema de salud pública.

La Organización Mundial de la Salud (OMS) ha señalado que anualmente alrededor de 16 millones de niñas entre 15 y 19 años dan a luz y en el caso de México en particular, la Encuesta Nacional de Salud y Nutrición (ENSANUT) muestra que en el año 2005 por cada mil mujeres embarazadas $30 \%$ correspondían a este grupo de edad y para el año 2011, se presenta un incremento importante del $37 \% .1$

Es por ello, que es necesario buscar estrategias, modalidades, técnicas y recursos atractivos para este segmento de la población vulnerable a esta problemática de salud, donde la incorporación de las TIC a los talleres de prevención del embarazo propicien que las nuevas generaciones, nativas de las tecnologías, le interese el tema y se motiven por aprender a cuidar su salud sexual y reproductiva.

Por lo anteriormente expuesto, la presente investigación tiene como objetivo implementar una estrategia didáctica basada en TIC, como herramientas que apoyen el proceso de enseñanzaaprendizaje en el taller de prevención del embarazo en adolescentes mediante una plataforma educativa.

\section{Referentes Teóricos}

\section{Las TIC en el Proceso de Enseñanza-Aprendizaje}

López y Fernández (2012) realizaron un estudio de investigación cualitativo con el objetivo de describir una experiencia de innovación en la enseñanza universitaria, con presentaciones educativas interactivas sobre los principales ámbitos de intervención socioeducativa del trabajador social con aplicaciones de software. 2

Los principales resultados obtenidos en este trabajo plantean que el uso de las TIC en los procesos de enseñanza-aprendizaje procuran más ventajas que inconvenientes, al favorecer el desarrollo de competencias necesarias para su posterior incorporación en el mundo laboral: trabajo en equipo, fomento de habilidades en relaciones interpersonales y el desarrollo de la autonomía.

Ferro, Martínez y Otero (2009) analiza las ventajas del uso de las TIC como recurso didáctico. Las conclusiones de este trabajo manifiestan que las principales ventajas son la ruptura de las barreras espacio-temporales, la posibilidad que ofrecen de interacción con la información y lo útil que resultan como herramientas de apoyo al aprendizaje. Por el contrario, la ventaja menos valorada ha sido el ahorro de tiempo que el profesor podría dedicar a otras tareas. 3

Por otro lado, Poblete (2008), en su investigación denominada "Uso de estrategias para mejorar la ortografía en los alumnos de tercer grado de nivel primaria" de la Universidad Nacional Autónoma de México, se plasman cada una de las cosas que abarcan las estrategias didácticas y la ortografía. La hipótesis se comprobó en tan solo diez sesiones, los niños demostraron un gran cambio en comparación de lo que se evaluó al principio. La autora propone que al trabajar con estrategias didácticas es importante dar a conocer su significado y por consiguiente que se seleccionen las estrategias adecuadas para el grupo y el tema que se explicará. 4
Es importante resaltar que siempre que se incorporen las TIC en las estrategias didácticas utilizadas en el proceso de enseñanzaaprendizaje es necesario considerar el modelo de diseño instruccional y la plataforma educativa a emplear.

El diseño instruccional es la organización del conocimiento, de los materiales didácticos y medios, es la arquitectura del aprendizaje porque permite al docente concebir la estructura de un curso o taller y la relación de cada elemento. 5

El propósito del diseño instruccional es proporcionar las condiciones necesarias para que el estudiante construya su propio conocimiento. 6

Los diseños instruccionales dan lugar a los modelos de diseño instruccional, los cuales son una guía para implementar los ambientes virtuales de aprendizaje.

En esta investigación se selecciona el modelo de diseño instruccional de Jerold Kemp y no otro porque se adecua al contexto, a los sujetos (adolescentes) bajo la teoría del aprendizaje constructivista. Los estudiantes son nativos de la tecnología pero no la utilizan en la vida diaria con fines educativos, por lo que el docente aprovecha estas condiciones para motivar y despertar el interés en ellos en la prevención del embarazo.

El modelo instruccional de Jerold Kemp tiene más en cuenta al estudiante permitiéndole el asumir un rol activo dentro del proceso que favorece la construcción de su propio aprendizaje. 7. Se basa en diez puntos básicos: Necesidades del estudiante, empleo de producto y propósito, características de los estudiantes, análisis de tareas, objetivos instruccionales, actividades de enseñanza, recursos instrucciones, servicios de apoyo, evaluación del aprendizaje y por último la Pre-Prueba. Por otra parte, la plataforma educativa es un Sistema de Gestión del Aprendizaje (SGA) en el cual se aloja los materiales didácticos digitales diseñados y se utilizan otras herramientas que permiten interactuar, comunicar y evaluar los aprendizajes de los alumnos.

En este trabajo se selecciona la plataforma educativa NEO porque es un sistema gratuito, no se tiene que descargar o instalar ninguna aplicación para su funcionamiento, además se puede registrar con facilidad. Cada profesor o institución educativa recibe un portal propio para personalizar y está disponible en español, las 24 horas del día, durante todo el año, es de fácil acceso para los estudiantes, inclusive a los padres de familia si quieren corroborar el aprendizaje de sus hijos.

\section{Estado del Arte}

Las estrategias didácticas son los métodos y técnicas que se utilizan para conseguir o lograr un objetivo. Las mismas son categorías de la didáctica como también los son: los objetivos, los contenidos, recursos didácticos, las formas de organización de la actividad docente y la evaluación, en un tiempo y espacio dado. Es importante señalar que ninguna categoría didáctica es más importante una que otra, conque falle una en el proceso de enseñanza-aprendizaje no se alcanzaría la finalidad educativa propuesta. 8

Al revisar la literatura científica relacionada con el uso de las TIC en estrategias didácticas se ha encontrado que en América Latina, uno de los países más avanzados en cuestión de educación y tecnología es Argentina, tal es el caso de la Facultad Latinoamericana de Ciencias Sociales (FLACSO), desde sus comienzos en 1974 
Desde las distintas unidades académicas, la FLACSO se ha dedicado a analizar la realidad regional desde una perspectiva latinoamericana, realizando un aporte significativo en el desarrollo de las ciencias sociales y siendo el primer centro de formación de posgrado de ese campo en Argentina. Tiene una intervención exitosa en el posgrado virtual, con el mismo nivel de excelencia que ofrece la FLACSO en su sede académica presencial de Buenos Aires.

En México comienza a impactar significativamente, los aportes de los estudios que dan cuenta de los debates actuales sobre los diversos temas sociales prioritarios para América Latina. Incluyendo a quienes residen en lugares muy distantes de donde ese conocimiento se produce, los diversos posgrados virtuales ponen a disposición de los estudiantes los conocimientos procedentes de diversas ciencias sociales, así como una perspectiva interdisciplinaria que incorpora renovados enfoques teóricos, sólidos modelos y experiencias de investigación.

Desde 2004, la FLACSO Virtual utiliza Software Libre para la construcción de su plataforma de educación en línea. Puntualmente, la plataforma Moodle, compuesta por diferentes módulos y orientada al armado de comunidades educativas.

Por otra parte, en México los cursos semipresenciales reconocidos en el área de salud, se otorgan por parte del Instituto Nacional de Salud Pública, está compuesta por profesionales especializados en las diversas disciplinas de la salud pública y de las ciencias sociales, por estudiantes que cursan programas académicos y por personal administrativo y técnico que apoya el cumplimiento de objetivos, programas, metas y proyectos que forman parte de la razón de ser de la institución. Está dirigido al público en general, este programa ofrece en los meses de julio y agosto cursos, diplomados y talleres en modalidad semipresencial. El curso en línea ofrece a los profesionales de la salud, un ciclo de videoconferencias sobre investigación en salud pública, con la finalidad de ampliar la cobertura de sus actividades de divulgación científica y educación continua.

Estos cursos tienen éxito, ya que cuentan con becas y pueden concursar de forma directa por medio de la Secretaría de Salud, tiene mayor énfasis en personas adultas profesionales, sí se está considerando el tema de prevención del embarazo, sin embargo no se otorga a los adolescentes.

Por último se aclara que no se han encontrado precedentes en la literatura científica revisada en las que se hayan utilizado SGA para talleres de prevención del embarazo.

\section{Metodología}

El procedimiento metodológico en la investigación para la implementación de la estrategia didáctica consiste en aplicar el modelo de diseño instruccional de Jerold Kemp, luego se selecciona la plataforma educativa NEO, en ambos casos las razones de la selección fueron explicadas con anterioridad en el presente documento, y las herramientas a emplear dentro del SGA, posteriormente se diseña el taller con modalidad semipresencial denominado: "Prevención del Embarazo en el Adolescente", seguidamente se lleva a cabo la etapa de evaluación. Por último se valida el impacto del curso a través de la aplicación de encuestas, de forma cualitativa y cuantitativa, a los sujetos involucrados.

Primeramente se aplica el modelo de diseño instruccional de Jerold Kemp, el cual se describe a continuación basado en sus diez puntos básicos.

1.- Necesidades del estudiante:
- De construir su identidad personal.

- De explorar, descubrir y experimentar.

- De renunciar a aspectos infantiles.

- De poner en práctica sus ideales.

- De tener información a los peligros que está expuesto.

- De tener confianza en sí mismo con reconocimiento de sus capacidades y limitaciones.

- De permanecer a un grupo de iguales.

- En la edad adolescente necesitan ser escuchados.

2.- Empleo de producto y propósito. Se centra en brindar a los estudiantes herramientas teóricas y metodológicas participando en el taller de prevención del embarazo en el adolescente, esta estrategia tendrá las siguientes vertientes:

- El diseño de material didáctico digital para la implementación en el aula de un taller preventivo.

- Capacitación y formación continua de los agentes involucrados (adolescentes) en la vida cotidiana, para fortalecer su preparación y el desarrollo de competencias vinculadas con el cuidado de la salud física, emocional y social.

- Formar estudiantes capaces de cuidar de sí mismos, de responsabilizarse de sus actos y de tomar decisiones libres para el autocuidado de su salud.

- Los temas a desarrollar serán infecciones de transmisión sexual, planificación familiar y factores de riesgo obstétrico.

- Fundamentalmente el estudiante desarrollará habilidades sociales e interpersonales, cognitivas que incluyan la toma de decisiones, el desarrollo del pensamiento crítico y el autocuidado de salud.

3.- Características de los estudiantes. Está dirigido a estudiantes adolescentes que cursen el nivel básico (secundaria), edad de 12 a 15 años, que dentro de su currícula se encuentre la asignatura de Biología Humana y pertenezcan a escuelas públicas con apego escolar.

4.- Análisis de tareas. Se determina el contenido del taller con los temas: infecciones de transmisión sexual, planificación familiar y factores de riesgo obstétrico.

5.- Objetivos instruccionales. Se asigna un objetivo por cada tema planteado.

6.- Actividades de enseñanza. Se diseña una estrategia didáctica que permita que el estudiante alcance los objetivos propuestos.

7.- Recursos instrucciones. Materiales didácticos digitales, audio y video, presentaciones digitales y mundo virtual.

8.- Servicios de apoyo. Se cuenta con personal capacitado, con equipos de cómputo y aula para llevar a cabo el taller. 9.- Evaluación del aprendizaje. Se evalúan los resultados obtenidos, el modelo permite realizar validación al final del curso, permitiendo realizar restructuración del curso y aula para llevar a cabo el taller.

10.- Pre-Prueba. Se lleva a cabo mediante la ejecución del taller educativo.

Posteriormente se utiliza la plataforma educativa NEO, la cual ofrece una comunidad de aprendizaje virtual que posibilita crear tu aula en línea, donde se pueden utilizar herramientas para fortalecer la enseñanza dentro del aula de clases o añadir recursos de propia creación o de otros.

Este SGA está compuesto por herramientas educativas, a continuación descritas: 
- Bienvenida: Se da a los alumnos que cursarán el taller, presentando el mapa del mismo y dando una breve descripción del tema a abordar.

- Noticias: Se publican acontecimientos relevantes en la actualidad dentro del medio referente al tema.

- Lecciones: Se dan a conocer a los estudiantes como parte del desarrollo de cada subtema para participar en discusiones o generar mayores conocimientos.

- Calendario: Se definen fechas para entrega de trabajos y tiempo del taller, el alumno podrá consultarlo para saber fecha de entrega de los trabajos.

- Foros: Se generan discusiones relativas a una serie de temas, los participante interactúan dando su opinión.

- Secciones: La herramienta de etiqueta tiene como objetivo representar las secciones que contemplan la plataforma educativa, correspondiente al inicio, desarrollo y cierre de los bloques, explicación de búsqueda de materiales didácticos digitales propuestos e invitaciones referente a dónde se sitúa una materia o actividad.

- Chat: Esta herramienta tiene como finalidad que el alumno se comunique con el docente por si en algún momento presenta dudas o cuestiones sobre un tema.

- Recursos: Almacena carpetas o archivos comprimidos, los cuales contienen materiales didácticos digitales, como son los objetos de aprendizaje, páginas web, presentaciones digitales, entre otros.

- Tareas: Se almacenan actividades realizadas por los alumnos que van generando en el transcurso del taller.

Después se diseñan los contenidos del taller, los cuales se muestran en forma de resumen en la Figura 1. Los contenidos están en función de articular todas las categorías didácticas antes mencionadas (objetivos, contenidos, métodos y estrategias, recursos didácticos, formas de organización, en este caso el Taller y por último la evaluación), en un tiempo determinado.

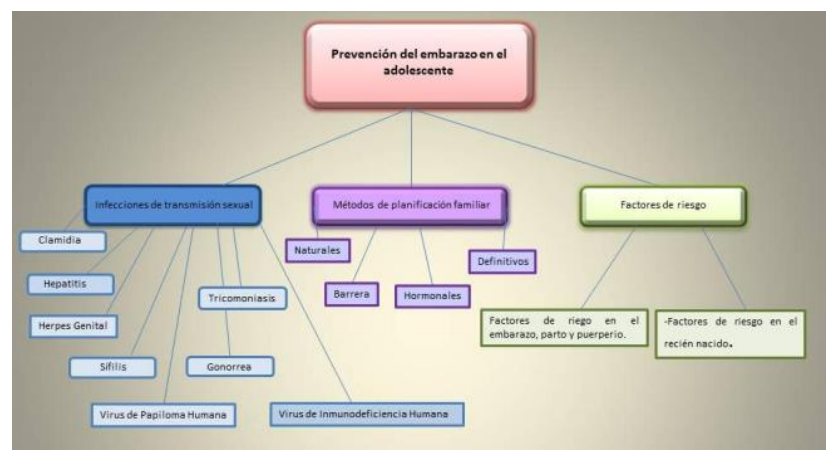

Figura 1. Mapa de los contenidos del Taller de prevención del embarazo en el adolescente

Los objetivos: general y específicos se enlistan a continuación. Objetivo general: Identificar el impacto del embarazo en la adolescencia con el propósito de interesar a los adolescentes para acudir a los servicios de salud donde se les brinde métodos de planificación familiar para prevenir embarazos no deseados y así evitar el contagio de infecciones de transmisión sexual que limiten su proyecto de vida.
Objetivo del bloque I: Indicar en qué consisten las infecciones de transmisión sexual que afectan la salud de los adolescentes a través de talleres educativos donde se dará a conocer la evolución, tratamiento y repercusiones para fomentar el autocuidado y el uso del preservativo.

Objetivo del bloque II: Describir los diferentes métodos de planificación familiar con la finalidad de que los adolescentes adopten el que mejor se adecue a su estilo de vida y de esta forma eviten embarazos no planeados que puedan interferir en su proyecto de vida.

Objetivo del bloque III: Explicar a través de talleres educativos los factores de riesgo obstétrico y sus consecuencias en el embarazo durante la adolescencia con la finalidad de identificar las complicaciones que implica un embarazo a temprana edad. El sistema de evaluación está compuesto por una heteroevaluación que equivale al $85 \%$, la cual ejecuta el docente, una coevaluación del $10 \%$ que se realiza entre los pares de estudiantes donde se valoran los aprendizajes logrados por sus compañeros y por último la autoevaluación que constituye un 5\%. Para todos los tipos de evaluación se emplean como instrumentos las listas de cotejo y rúbricas.

Se diseña una encuesta para la validación de la estrategia didáctica como instrumento de recolección de datos. Las preguntas combinan cualidades y cantidades a partir de responder al paradigma mixto de investigación. Se determina el tamaño de muestra representativa de la población, a través de fórmula matemática-estadística, dando como resultado 40 alumnos que participan en el taller de la secundaria objeto de estudio. El muestreo es aleatorio simple considerando adolescentes de los tres grados de secundaria del sector público.

\section{Análisis y Discusión de Resultados}

El cuestionario aplicado es confiable, se calcula el coeficiente alfa de Cronbach con el apoyo del SPSS para Windows, obteniendo un valor de 0.93 , lo cual evidencia que existe estabilidad y consistencia en las respuestas de los alumnos. Sí se aplica la encuesta a los mismos sujetos bajo las mismas condiciones se obtendrán resultados similares, así como se garantiza la validez porque el instrumento mide lo que realmente debe medir debido a que se involucran los indicadores establecidos en la metodología.

Al realizar la evaluación del aprendizaje con el uso de las TIC (heteroevaluación, coevaluación y autoevaluación) y emitir un juicio de valor los resultados de la calificación en un $90 \%$ están por encima de un $70 \%$ de aprovechamiento satisfactorio, no así cuando no se utilizan las TIC donde el $45 \%$ de los estudiantes logran calificaciones por debajo del $70 \%$.

Al comparar los resultados obtenidos en la evaluación del aprendizaje con los mismos sujetos, cuando recibían el taller de forma presencial, con respecto a cuándo lo reciben utilizando las TIC, se observa una diferencia significativa en las calificaciones de los alumnos, corroborado a través de una prueba de hipótesis estadística de igualdad de medias de tstudent, con respecto a la variable calificación obtenida en los aprendizajes del curso.

Este resultado evidencia la efectividad que se muestra para aprender con significado mediante el uso de las herramientas tecnológicas porque los alumnos obtienen mejores calificaciones, al aclarar las dudas entre pares y con el profesor con mayor facilidad y rapidez, la comprensión del contenido 
puede asimilarse de diversas formas, gracias a las tecnologías, con audios, videos, colores, formas, lo cual desarrolla y ejercita en el individuo la capacidad de abstracción y del pensamiento crítico.

De los 13 ítems que contempla el cuestionario aplicado para la validación de la propuesta de estrategia didáctica, se expone con énfasis las respuestas a la pregunta relacionada con el taller y el uso de las tecnologías. Ver Figura 2.

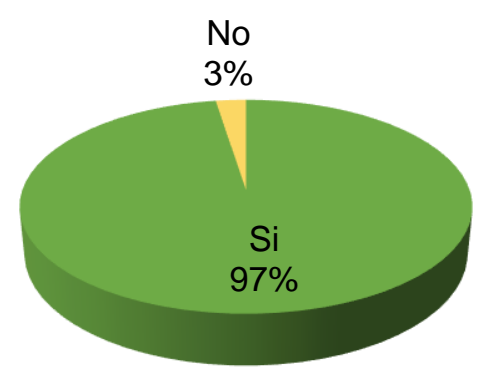

\section{Figura 2. Preferencia y utilidad por asistir al Taller: "Prevención del embarazo en el adolescente" con el uso de TIC.}

Las contestaciones evidencian que el $97 \%$ de los alumnos se sienten cómodos y motivados por asistir al taller con la incorporación de TIC debido a que ellos pueden comunicarse e interactuar con sus compañeros y el profesor ante cualquier duda, sugerencia y/o pregunta al respecto de cada tema abordado. Ellos tienen las habilidades tecnológicas para el manejo de herramientas de esta naturaleza porque son nativos de la tecnología digital y esto el profesor lo tiene a su favor porque lo puede canalizar con fines educativos.

El 3\% contesta a esta interrogante que no le gusta y no le confiere utilidad al taller con el uso de las TIC porque manifiestan que es necesario tener acceso a Internet y ellos en sus hogares no cuentan a veces ni con equipo de cómputo o dispositivo móvil, lo cual representa una desventaja para este sector de la población que por escasos recursos económicos no pueden disfrutar de esta posibilidad.

En los restantes 12 ítems las respuestas han sido bastante similares porque los alumnos en su inmensa mayoría, superior al $95 \%$, responden de manera afirmativa porque el uso de las TIC en el taller le propicia un ambiente de aprendizaje didáctico y divertido, donde ellos pueden acceder a la información, la retroalimentación y envío de tareas, así como evaluaciones desde cualquier lugar con acceso a Internet y en el momento que tengan el tiempo disponible cumpliendo con el horario y la fecha límite previamente establecida.

Los resultados obtenidos permiten que los estudiantes logren un aprendizaje con significado y útil para su vida futura producto a que logran tomar conciencia de la importancia de la prevención de embarazos en edades tempranas, tanto para el hombre como para la mujer, por lo que implica para ellos en su proyecto de vida.

También se toma en cuenta que ellos investigan y cuestionan las infecciones de transmisión sexual que existen, los métodos de prevención, las formas de planificación familiar, así como los factores de riesgo obstétrico que pueden llevar a la muerte del individuo o de su descendencia.

\section{Conclusiones}

Los resultados de la investigación demuestran que se fomenta la autonomía en el estudiante, logrando que los adolescentes participen activamente y dando su opinión acerca de los temas de prevención del embarazo.

El trabajo fuera del aula otorga flexibilidad de horario al alumno, en el momento oportuno desde su casa o cualquier lugar con acceso a Internet, él puede decidir acceder al taller de manera virtual y realizar sus actividades solicitadas por el profesor.

Los estudiantes ahora son capaces de tener nuevas experiencias de aprendizaje auténticas y significativas. Por ejemplo, la educación de hoy en día no se centra en los conceptos o hechos simplemente mostrados en los programas y planes de estudios. En su lugar, se trata del proceso de construcción de conexiones, como consecuencia, los alumnos adquieren una conciencia de la importancia y el valor de la comunicación.

Es válido resaltar que la temática abordada en el taller representa un impacto de manera positiva en la vida del adolescente, en sus relaciones personales y en el autocuidado de salud, pero que no debemos dejar de atender las necesidades de los alumnos del sector público que por no contar con los recursos económicos para acceder a equipos de cómputos y el Internet se encuentran en desventajas con los que tienen la posibilidad. Es por ello, que se deben crear estrategias como establecer horarios flexibles en los laboratorios de cómputos de las escuelas, con acceso a la red de redes para que se les facilite la conexión y el desarrollo del taller a los estudiantes en condiciones vulnerables desde el punto de vista económico.

\section{Referencias}

[1] Montejano, C. (2013). El embarazo en Adolescentes. Marco Teórico Conceptual, Políticas Públicas, Derecho Comparado, México, D.F.

[2] López, E., \& Fernández, R.M. (2012). Implicaciones de las TIC en el ámbito socio-educativo y de servicios sociales: una experiencia universitaria de innovación y desarrollo docente con tecnología 2.0. Sevilla, España.

[3] Ferro, C., Martínez A. \& Otero, M.C. (2009). Ventajas del uso de las TIC en el proceso de enseñanza-aprendizaje desde la óptica de los docentes. Revista Electrónica de Tecnología Educativa, 29.

[4] Poblete. (2008). Uso de estrategias para mejorar la ortografía en los alumnos de tercer grado de nivel primaria. México, D.F.

[5] Kemp, J. (1997). Instrucccional Desing: A plan for unit and course development.

[6] Díaz Barriga, F.; Hernández, G. \& Rigo, M.A. (2009). Aprender y enseñar con TIC en educación superior. Contribuciones del socioconstructivismo, UNAM: México. México: Mc Graw Hill.

[7] Guamán, M.A.(Julio de 2015). Modelo de Diseño Instruccional de Jerold Kemp. Quito, Ecuador.

[8] Díaz Barriga, F. \& Hernández, G. (2010). Estrategias docentes para un aprendizaje significativo, Una interpretación constructivista. México: Mc Graw Hill. 